\title{
ERRATUM
}

\section{Predictors of Neuropsychological Improvement Following Cognitive Rehabilitation in Patients with Gliomas-Erratum}

Karin Gehring, Neil K. Aaronson, Chad M. Gundy, Martin J.B. Taphoorn, AND Margriet M. Sitskoorn

doi:10.1017/S1355617710001530, Published online by Cambridge University Press on 21 December, 2010.

The paper by Gehring et al. (2011) has errors in the article that should be brought to the attention of readers. The instances are listed with their page numbers along with the errant and correct copy:

Page 4, Table 3 Note: errant copy—Number and percent of improves; correct copy—Number and percent of improvers

Page 5, Right column, three lines under the head "RCI Results ...": errant copy $-p=.000$; correct copy-p<.001

Page 6, Left column, under the head "Logistic Regression Analyses":

(a) last line of the first paragraph: errant copy $-p=.000$; correct copy $-p<.001$

(b) first line of the third paragraph: errant copy $-p=.000$; correct copy $-p<.001$

Right column,

(a) second paragraph, first line: errant copy $-p=.000$; correct copy $-p<.001$

(b) second paragraph, lines 8 and 9: errant copy-The odds for...; correct copy-The odds of...

Page 8, Left column, second paragraph, line 10: errant copy $-p=.000 ;$ correct copy $-p<.001$.

NOTE: These errors were corrected before JINS 17:2, March 2011, was sent to press and do not appear in print. In addition, the page numbers listed above represent the temporary numbers that appeared in the initial online version of Gehring et al. (2011).

Cambridge University Press and the Editor regret the inconvenience that these errors may have caused. 